\title{
FAST SPATIALLY CONTROLLABLE 2D/3D TEXTURE SYNTHESIS AND MORPHING FOR MULTIPLE INPUT TEXTURES
}

\author{
Felix Manke and Burkhard Wünsche \\ Graphics Group, Department of Computer Science, University of Auckland, Private Bag 92019, Auckland, New Zealand \\ FelixManke@gmx.de,burkhard@cs.auckland.ac.nz
}

Keywords: Exemplar-based texture synthesis, Texture morphing, Solid texture, Appearance space.

\begin{abstract}
Texture synthesis and morphing are important techniques for efficiently creating realistic textures used in scientific and entertainment applications. In this paper we present a novel fast algorithm for multi-dimensional texture synthesis and morphing that is especially suitable for parallel architectures such as GPUs or direct volume rendering (DVR) hardware. Our proposed solution generalizes the synthesis process to support higher than three-dimensional synthesis and morphing. We introduce several improvements to previous 2D synthesis algorithms, such as new appearance space attributes and an improved jitter function. We then modify the synthesis algorithm to use it for texture morphing which can be applied to arbitrary many 2D input textures and can be spatially controlled using weight maps. Our results suggest that the algorithm produces higher quality textures than alternative algorithms with similar speed. Compared to higher quality texture synthesis algorithms, our solution is considerablly faster and allows the synthesis of additional channels without affecting the running time of the synthesis at all. The method is easily extended to allow fast 3D synthesis and as another novel contribution we show several examples for morphed solid 3D textures. Overall the presented technique provides an excellent trade-off between speed and quality, is highly flexible, allows the use of arbitrary channels such as transparencies and displacement maps, can be extended to arbitrary dimensions and is suitable for a GPU-implementation.
\end{abstract}

\section{INTRODUCTION}

Texture mapping is one of the most important techniques to increase the realism of a 3D scene by providing fine surface details. Exemplar-based 2D texture synthesis is a powerful tool to generate large textures from small input examples. Texture morphing as an extension creates coherent transitions between entirely different materials with a quality and flexibility that cannot be achieved using simple blending techniques. The applications of texture morphing are manifold and include terrain rendering, scientific visualization, the creation of transitions in animal fur and between biomedical or geological materials, and the simulation of aging processes.

3D solid textures have the advantage that, in contrast to 2D textures, objects can be "carved" out of a 3D material resulting in more realistic results. Since the acquisition of 3D textures is difficult, the synthesis and morphing of solid textures from 2D exemplars is very important. However, the task is extremely challenging and usually requires long computation times.
In this paper, we present a new fast algorithm for exemplar-based 2D texture morphing and higher-dimensional texture synthesis and morphing. Our texture morphing algorithm is based on Lefebvre and Hoppe's pixel-based texture synthesis algorithm (Lefebvre and Hoppe, 2005), a real-time approach implemented on the GPU by utilizing the parallel synthesis scheme proposed by L. Wei (Wei, 2003).

Because of the close relationship we will give a brief summary of the original synthesis algorithm in section 3 before discussing the extensions we made to support texture morphing of arbitrary many input exemplars in 2D (section 4) and 3D (section 5). In section 6 we discuss our results and conclude with an outlook on future research in section 7 .

\section{RELATED WORK}

Texture synthesis and texture morphing are closely related fields in which numerous different algorithms 
have been proposed. Procedural techniques for both 2D and 3D texture synthesis (Perlin, 1985; Turk, 1991; Witkin and Kass, 1991; Worley, 1996) proofed to be hard to control and, compared to exemplar-based methods, were limited in the types of materials that can be modeled. Parametric exemplar-based methods, as proposed in (Heeger and Bergen, 1995; De Bonet, 1997; Bar-Joseph et al., 2001), rely on models of global statistical properties which serve as constraint function while matching statistics of the input and target texture. Though extensions for 3D synthesis have been made (Heeger and Bergen, 1995; Dischler et al., 1998), parametric models are usually only successful in synthesizing homogeneous and stochastic exemplars. Mixing properties of different textures is possible, but for texture morphing not enough spatial control is offered. Patch-based methods paste random patches of the exemplar into the output texture and optimize the transitions between the overlapping patches (Praun et al., 2000; Efros and Freeman, 2001; Kwatra et al., 2003). While these methods could probably be extended to use 3D texture patches as input, there is no straightforward way to use them to generate $3 \mathrm{D}$ textures from a $2 \mathrm{D}$ input. Indeed, we believe it is questionable whether such techniques can be used to create texture morphing of acceptable quality at all (though Kwatra et al. placed flower textures onto a grass texture and optimized the grass seams (Kwatra et al., 2003)). In contrast, by processing one pixel at a time pixel-based methods (Efros and Leung, 1999; Wei and Levoy, 2000; Ashikhmin, 2001; Lefebvre and Hoppe, 2005) offer a control that is fine enough to allow texture synthesis and morphing in $2 \mathrm{D}$ and $3 \mathrm{D}$ at a high quality. A successful 3D synthesis has been shown by L. Wei (Wei, 2002; Wei, 2003). Finally, optimization-based approaches use the local similarity measures of pixel neighborhoods to define a global texture energy function that is minimized (Kwatra et al., 2005). Recently, Kopf et al. demonstrated that energy-based methods can be used for 3D synthesis (Kopf et al., 2007), though the synthesis times are with up to 90 minutes rather slow and a GPU implementation non-trivial. Another specialized solution for synthesizing 3D composite materials based on stereology theories (the analysis of 2D cross-sections of $3 \mathrm{D}$ volumes) has been proposed by Jagnow et al. (Jagnow et al., 2004).

Algorithms specifically for 2D texture morphing have also been developed. L. Wei used his pixelbased method to create transitions between two exemplars (Wei, 2003). However, the synthesis quality decreased significantly. Liu et al. proposed a patternbased approach that uses ideas of image morphing to generate metamorphosis sequences (Liu et al., 2002).
Both Zhang et al. and Tonietto and Walter used texton maps to support a pixel-based texture morphing (Zhang et al., 2003; Tonietto and Walter, 2005). Unfortunately, all three publications show results only for very similar input exemplars. Matusik et al. utilized a simplical complex model to build a neighborhood graph of input exemplars (Matusik et al., 2005). Though examples with several input textures are given, the approach relies on a texture database and is explicitly designed for similar textures only. To our knowledge, texture morphing in three dimensions using exemplars of very different irregularly textured materials has not been shown by anyone before.

\section{LEFEBVRE AND HOPPE'S 2D TEXTURE SYNTHESIS}

As most pixel-based methods, the algorithm proposed in (Lefebvre and Hoppe, 2005; Lefebvre and Hoppe, 2006) performs an iterative optimization to minimize the difference of the synthesis result to the original exemplar, where the distance is measured using the sum of squared differences (SSD) of local neighborhoods. A standard multi-resolution approach is pursued by computing a Gaussian pyramid $E$ of the exemplar and creating an "empty" pyramid $S$ for the synthesis result. The synthesis is performed from the coarsest to the finest resolution, first establishing low frequencies and then defining the fine details.

A key difference of the algorithm to other methods is the fact that $S$ does not store image colors, but pixel coordinates into the exemplar $E$ in order to enable a GPU implementation. To pass the synthesis result of a coarse level $S_{i-1}$ to a finer level $S_{i}$ an upsampling of the coordinates is performed that distributes the value of $S_{i-1}(P)$ (that is, a coordinate into $E_{i}$ ) to four child pixels in $S_{i}$. In the correction phase, the synthesis error is minimized by searching the pixel coordinate $Q$ with the best-matching local neighborhood $N_{E_{i}}(Q)$ in the exemplar for the neighborhood $N_{S_{i}}(P)$ around $S_{i}(P)$. The correction phase is accelerated using subpasses, each of which optimizes only selected pixels, and $k$-coherence search (Tong et al., 2002) based on pre-computed candidate sets.

The texture synthesis can greatly benefit by using an appearance space (Lefebvre and Hoppe, 2006), where pixels encode texture characteristics in addition to color. The high-dimensional appearance vectors are projected into a low-dimensional space defined by the first $n$ principal components obtained from a principal component analysis (PCA). In tests we found that usually more than $95 \%$ of the total variance of an exemplar is explained by the first 8 components. 


\section{OUR 2D TEXTURE MORPHING ALGORITHM}

When dealing with texture morphing we have to synthesize a texture based on several input exemplars. The result should reflect the nature of all exemplars, though the influence of each input can vary spatially. To control the spatial influence of each of the $m$ exemplars, we use scalar weight maps of the size of the synthesized texture $S$. Each weight map $W^{j}$ encodes the weight of the exemplar $E^{j}$ per position $P \in S$. To ensure a correct morphing, we normalize the weight maps so that $\sum_{j=1}^{m} W^{j}(P)=1$. Note that, when specifying only one input exemplar, our algorithm behaves like a standard texture synthesis algorithm.

In the following, we will discuss the extensions we made for every single step of the original synthesis algorithm. Because the coordinate upsampling remains unchanged and is performed on each $S_{i}^{j}$ individually, we do not include it in the discussion. Note that the modifications necessary for texture morphing still allow an implementation on parallel architectures.

\subsection{Initialization}

The initialization of the exemplars themselves remains unchanged, because they are independent from each other. For every weight map $W^{j}$ that is associated with each exemplar, we additionally compute a Gaussian pyramid. Because the algorithm is based on manipulating exemplar coordinates rather than colors (and coordinates cannot be averaged or merged), we need a separate synthesis pyramid $S^{j}$ for every exemplar. Instead of initializing $S_{-1}^{j}$ with zero coordinates, we find the following initialization more intuitive (where $s_{e^{j}}$ is the size of $E^{j}$ ):

$$
S_{-1}(P)=P \bmod s_{e^{j}},
$$

as this better reflects how the algorithm proceeds, especially when only a few pyramid levels are used.

\subsection{Coordinate Jitter}

The coordinate jitter is also independent for each exemplar. We propose a more intuitive jitter function that has a more uniform distribution than the function proposed by (Lefebvre and Hoppe, 2005):

$$
\begin{aligned}
J_{i}(P)=\left\lfloor j+\left(\begin{array}{l}
k_{x} \\
k_{y}
\end{array}\right)\right\rfloor, \text { where } \\
j=\mathfrak{H}(P) \cdot \operatorname{lerp}\left(0.5,1, r_{i}\right), \\
k_{x \mid y}= \begin{cases}\operatorname{lerp}\left(0.5,2 / 3, r_{i}\right) & \text { if } j_{x \mid y} \geq 0 \\
1-\operatorname{lerp}\left(0.5,2 / 3, r_{i}\right) & \text { otherwise, }\end{cases} \\
\operatorname{lerp}(a, b, t)=a+t(b-a) .
\end{aligned}
$$

\subsection{Correction Phase}

In the correction phase the best-matching pixel for a synthesis pixel $P$ is searched in the exemplar. However, now $S_{i}(P)$ must represent all input exemplars, weighted according to the weights $W_{i}^{j}(P)$. For the original 2D synthesis algorithm the neighborhood of $P$ in the synthesis level $S_{i}$ can be defined as the set:

$$
N_{S_{i}}(P)=\left\{E_{i}(Q) \mid Q=S_{i}\left(P+\Delta_{N}\right)\right\}, \Delta_{N} \in \mathcal{N},
$$

where $\Delta_{N}$ takes the values of offset vectors from the neighborhood's center to all pixels in the neighborhood as defined by the set $\mathcal{N}$ of all offsets:

$$
\mathcal{N}=\{\delta \mid(P+\delta) \in N(P)\} .
$$

For the texture morphing of multiple exemplars, we extend the neighborhood gathering as follows:

$$
\begin{aligned}
& N_{S_{i}}(P)=\left\{C\left(i, P+\Delta_{N}\right)\right\}, \Delta_{N} \in \mathcal{N}, \\
& C(i, X)=\sum_{j=1}^{m} W_{i}^{j}(X) \cdot E_{i}^{j}(Q), Q=S_{i}^{j}(X) .
\end{aligned}
$$

$C(i, X)$ the synthesized color in level $i$ at location $X$ as the weighted average of all exemplar levels. Given the synthesis neighborhood $N_{S_{i}}(P)$ the best-matching pixel is searched for in each of the exemplars $E_{i}^{j}$ and $S_{i}^{j}(P)$ where $N_{S_{i}}(P)$ is the same for all $m$ synthesis levels $S_{i}^{j}(P)$ :

$$
S_{i}(P)=\underset{Q \in E_{i}}{\operatorname{argmin}} \operatorname{SSD}\left(N_{S_{i}}(P), N_{E_{i}}(Q)\right) .
$$

\section{3D TEXTURE SYNTHESIS AND MORPHING}

In the following, we will discuss how to generate textures with an additional spatial dimension and morph between different materials. As illustrated in figure 1, several input exemplars are considered as being different views of the solid texture cube that is to be synthesized (Wei, 2002; Kopf et al., 2007). During the synthesis, the algorithm tries to generate a $3 \mathrm{D}$ texture that reflects the characteristics of all views. The close relationship to $2 \mathrm{D}$ texture morphing in terms of the use of multiple exemplars was already mentioned by L. Wei (Wei, 2002). To create smooth transitions between entirely different materials within the solid texture cube, exemplar views need to be specified for each material. For 3D morphing the weight maps are used as in the 2D morphing to define the spatial influence of the materials. Our algorithm gives a unified tool for supporting 3D synthesis or morphing at the same time. 


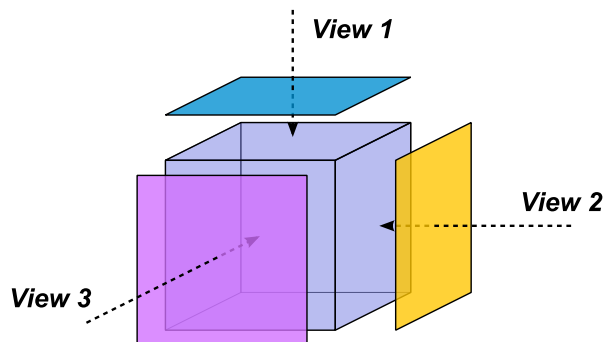

Figure 1: Several 2D exemplars define different views of the solid texture being synthesized. Adopted from (Wei, 2002).

It is not always necessary or appropriate to specify all three exemplars. Sometimes, for example when a material exhibits dominant directional features, it is better to define only two views (Wei, 2002).

The jitter step is not affected by our modifications, because the coordinates stored in a synthesis level $S_{i}^{j}(P)$ are still defined in $\mathbb{R}^{2}$. A minor difference is that the jitter function $J_{i}(P)$ and the hash function $\mathfrak{H}(P)$ now take 3D coordinates as input argument.

\subsection{Initialization}

In contrast to the $2 \mathrm{D}$ morphing, the synthesis pyramids $S^{j}$ and the weight maps $W^{j}$ are solid texture cubes. In $S^{j}$ each voxel stores a 2D coordinate into the corresponding exemplar $E^{j}$. Because $E^{j}$ represents only one particular view onto the solid target texture, the initialization of $S_{-1}^{j}$ is modified in the following way:

$$
S_{-1}^{j}(P)=P_{u, v} \bmod s_{e^{j}},
$$

where $u$ and $v$ denote the two components of $P \in \mathbb{R}^{3}$ to which $E^{j}$ is parallel.

\subsection{Coordinate Upsampling}

The coordinate upsampling cannot simply be extended by an additional dimension, because the synthesis pyramids still store $2 \mathrm{D}$ coordinates. Thus, we apply the 2D upsampling for every second slice that is oriented parallel to the exemplar view and duplicate the result for each subsequent slice:

$$
\begin{array}{r}
S_{i}^{j}\left(2 \cdot P_{u, v \mid w}+\Delta_{u, v}\right)= \\
\left(2 \cdot S_{i-1}^{j}\left(2 \cdot P_{u, v \mid(w+1)}+\Delta_{U}\right) \bmod S_{e^{j}},\right.
\end{array}
$$

where $w$ is even $(w \bmod 2=0)$ and depicts the component of $P$ that is orthogonal to the view. $\Delta_{u, v}$ describes a 3D vector with the same value in the $u$ - and $v$-component as $\Delta_{U}$ and $w=0$.
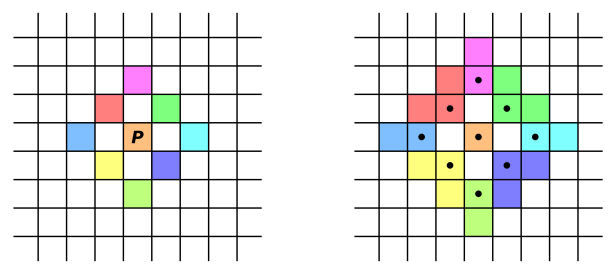

Figure 2: Left: Pixels used in our half-reduced neighborhood. On the right, all pixels are shown that are included when computing the averaged values of the neighborhood.

\subsection{Correction Phase}

When performing neighborhood-matching during the correction phase we have to deal with the problem that neighborhoods around voxels in a 3D synthesis pyramid have to be compared with neighborhoods of pixels of a 2D input exemplar. As a solution we take advantage of the fact that the exemplars are oriented and stand orthogonal to one of the principal axes of the solid cube that is synthesized. Since the exemplar represents only the view in this direction, the synthesis pyramid for this particular exemplar also only needs to reflect the exemplar in the same direction. Thus, we can align the $2 \mathrm{D}$ neighborhoods $N_{S_{i}}(P)$ to stand parallel to the exemplar, as it has also been proposed in (Wei, 2002; Kopf et al., 2007).

In consequence, we need to introduce several synthesis neighborhoods $N_{S_{i} \mid u, v}(P)$, one for each possible orientation of exemplars. Note that, as in the 2D morphing algorithm, $N_{S_{i} \mid u, v}(P)$ is a merged neighborhood that needs to represent all exemplars. Our definition in equation 2 is also valid for the $3 \mathrm{D}$ synthesis, except that $P \in S_{i}^{j}$ is now defined in $\mathbb{R}^{3}$. We therefore modify the definition to support oriented neighborhood gathering in 3D solid textures:

$$
N_{S_{i} \mid u, v}(P)=\left\{C\left(i, P+\Delta_{N \mid u, v}\right)\right\}, \quad \Delta_{N \mid u, v} \in \mathcal{N}_{u, v},
$$

where $\Delta_{N \mid u, v}$ gives the neighborhood offsets parallel to the current view (and the $w$-component set to 0 ). Notice the similarity to the upsampling step, which also depends on the view's orientation.

Interleaved correction for an improved convergence using sub-passes is still possible in 3D synthesis and morphing. However, because of the additional dimension we now have to define eight sub-passes as a pattern in a $2^{3}$ cube.

\subsection{A New Neighborhood}

As discussed in section 3, the synthesis in the reduced appearance space makes it possible to use a very compact neighborhood consisting of only 4 corner pixels. 

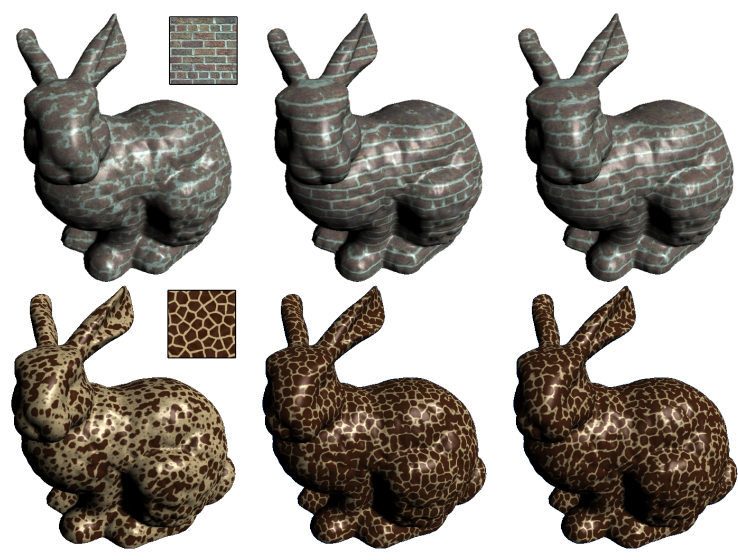

Figure 3: Comparison of different neighborhoods used during the correction phase of the 3D texture synthesis. First column: Reduced four-pixel neighborhood. Second column: Full $5 \times 5$ neighborhood consisting of 25 pixels. Third column: Our new half-reduced neighborhood consisting of 9 pixels.

However, 3D texture synthesis is a much more challenging problem. The algorithm generally has to deal with little information that is available for generating a solid texture out of only 2D exemplars. We found that the reduced neighborhood is not capable of preserving the features of the input exemplars. Much better results can be achieved using a full $5 \times 5$ or even $7 \times 7$ neighborhood - of course at the expense of speed.

To improve the synthesis while keeping the computation time low we propose a new "half-reduced" neighborhood. The layout is shown in figure 2 (left). We still average several pixel values to compute the values for the individual neighborhood values (shown on the right of the figure). Note that the new neighborhood, consisting of 9 points, is a superset of the neighborhood proposed in (Lefebvre and Hoppe, 2006).

Figure 3 shows a comparison of the results using different neighborhoods for 3D synthesis. Clearly, our new neighborhood is much better capable of preserving feature coherence than the original four-pixel neighborhood, which fails to produce acceptable results. The new neighborhood can also be used for $2 \mathrm{D}$ texture synthesis and morphing. We found that 2D morphing results are improved significantly for exemplars with semantic features.

\subsection{Synthesis and Morphing of Additional Channels}

The synthesis and morphing based on texture coordinates makes it possible to restore the exact location in the original exemplar for each pixel in the synthesized texture. Instead of a color image, the result of the syn- thesis is a map of texture coordinates that is used to sample the exemplars and output the final image.

Using this map of texture coordinates, we are able to sample arbitrary input images, and not only the color exemplar. Hence, additional channels — like alpha channels, displacement maps, specularity maps, etc. - can be synthesized without affecting the performance of the synthesis/morphing at all. This is an advantage over other methods that do not keep track of the original pixel locations in the input exemplars.

\section{RESULTS}

We implemented our algorithm using $\mathrm{C}++$ and execute it on the CPU in order to facilitate experimentation and integration into existing biomedical visualization software. As exemplars we used $64 \times 64$ or $128 \times 128$ pixel textures. For the $2 \mathrm{D}$ morphing, the target size is $512 \times 512$. Our generated solid textures have $128^{3}$ or $256^{3}$ voxels. We used the appearance space attributes as discussed in section 3 and projected the 150-dimensional vectors onto the first eight components using the PCA implementation from (Intel ${ }^{\circledR}$ Corporation, 2008). We performed two full correction passes per synthesis level. For the 2D outputs we used the reduced or our half-reduced neighborhood, for 3D synthesis and morphing the half-reduced or a full $5 \times 5$ neighborhood.

Figure 4 shows 2D morphing results with two exemplars to demonstrate how the transition between structures is generated. Note how the algorithm gradually defines a coherent transition of features and morphs between them, even if the exemplars' features are extremely different. Figure 5 depicts morphings with several exemplars and complex weight maps.

Examples of our 3D synthesis, including one with additional channels for displacement mapping, and morphing results are given in figures 6 and 7, respectively. As can be seen, the generated 3D solid textures coherently reflect the characteristics of the materials. However, a smoothing of the fine details can be observed - a problem that is common in solid texture synthesis algorithms (compare for example to (Wei, 2003; Kopf et al., 2007)).

Using one core of a $2.13 \mathrm{GHz}$ Intel ${ }^{\circledR}$ Core $^{\mathrm{TM}} 2$ Duo CPU with 2 GB RAM the 2D morphing using two exemplars and a reduced neighborhood takes only less than 12 seconds on average. With our halfreduced neighborhood the timings are still below 17 seconds and examples with three and four exemplars needed less than 18 and 25 seconds, respectively, for the morphing with a reduced neighborhood.

For $3 \mathrm{D}$ synthesis, even with a full $7 \times 7$ neigh- 

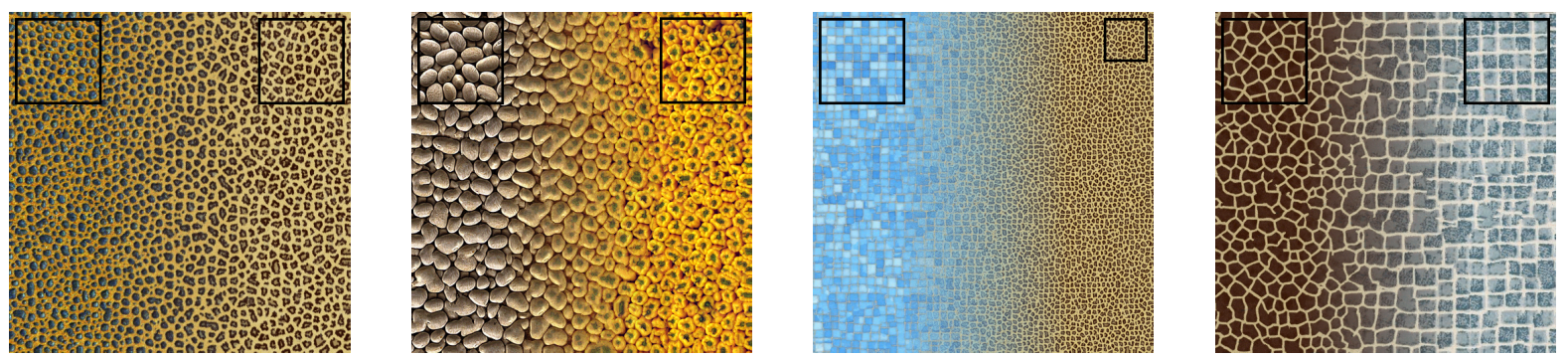

Figure 4: 2D morphing results using two input exemplars and linear weight maps. The examples illustrate how the morphing algorithm finds coherent transitions between the features.
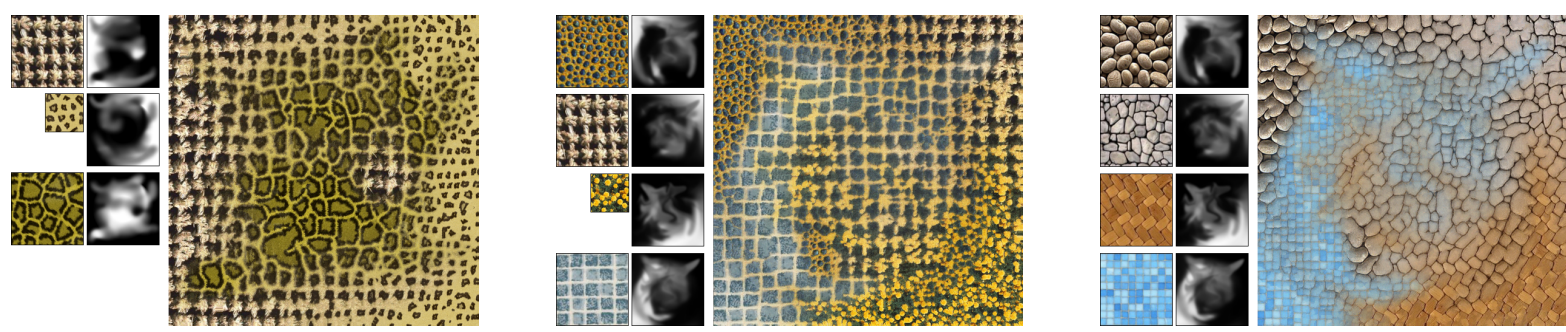

Figure 5: 2D morphing results using three and four input exemplars and complex weight maps. Note that the weight maps are normalized and of the same size as the synthesized target texture.
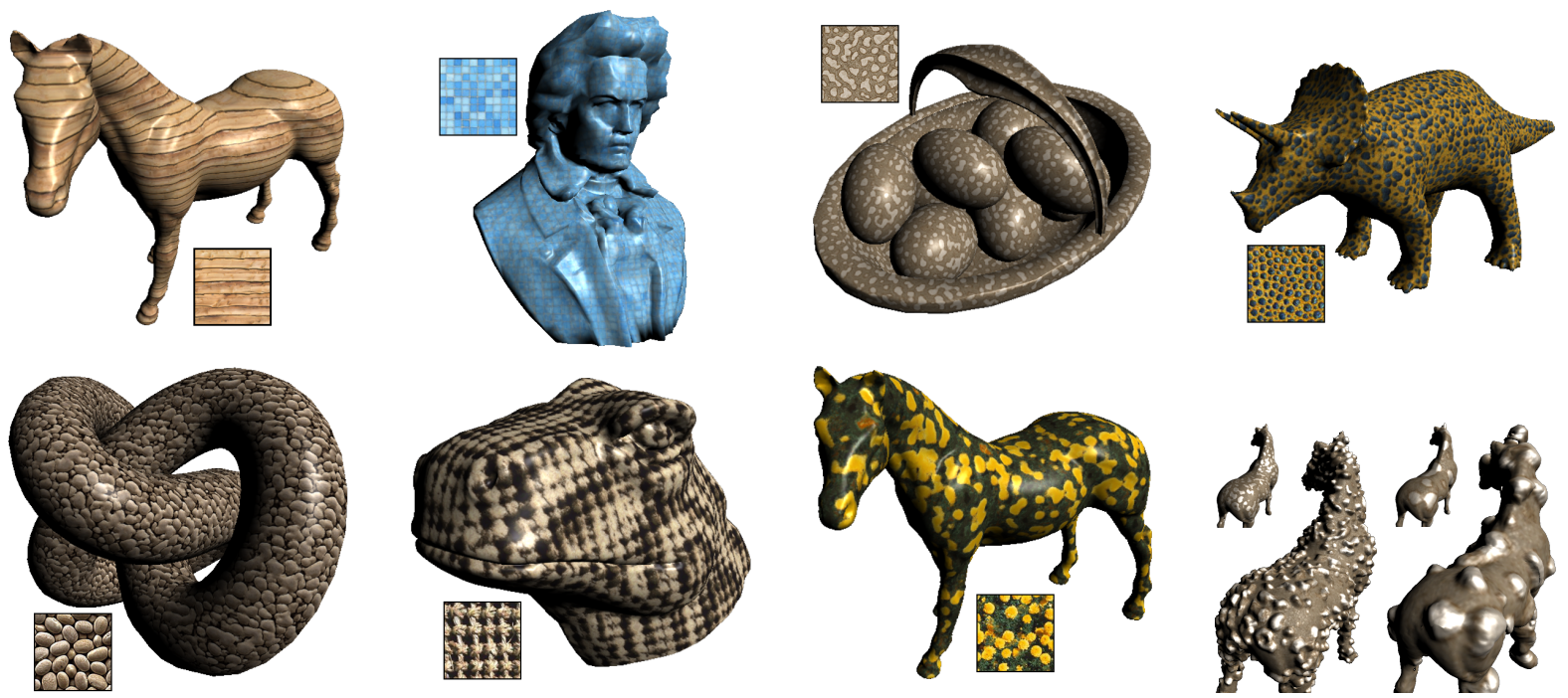

Figure 6: 3D texture synthesis results. The generated solid textures have been used to render different 3D geometries. Bottomright: Intensity values used as additional channel for displacement mapping.
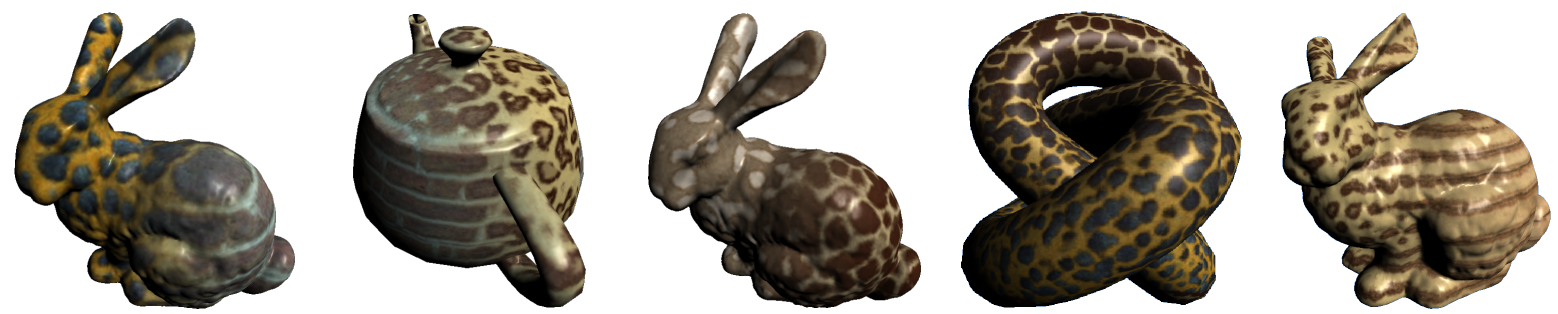

Figure 7: 3D morphing results using two exemplars and linear weight maps. The last two examples use a morphed texture of size $256^{3}$. 
borhood (which we almost never use in practice) our algorithm needs no more than 15 minutes to synthesize a $128^{3}$ solid texture cube, and 3D morphing with twice as many exemplar views takes less than 30 minutes. The half-reduced neighborhood performs with little more than 5 minutes for 3D synthesis and about 12 minutes for 3D morphing very fast while producing high-quality results. For morphing solid textures with a resolution of $256^{3}$ voxels, our algorithm needs between 110 minutes (half-reduced neighborhood) and 140 minutes (full $5 \times 5$ neighborhood). Note that doubling the target resolution leads to eight times as many voxels in the solid cube. With an implementation on the GPU we would expect a significant performance boost, possibly by several orders of magnitude.

Our algorithm has some limitations. As most pixel-based approaches the synthesis and morphing sometimes fails for textures with large features or where the features have a semantic meaning to humans. Problems also occur with near stochastic textures such as clouds and slightly crumbled paper. Textures with features of very different scale represent a particular problem for the morphing, because no common structures can be found that could be morphed into each other.

\subsection{Comparison with Kopf et al.'s Algorithm}

In figure 8 , we compare the synthesis quality of our proposed algorithm with results of the algorithm based on energy minimization recently presented by (Kopf et al., 2007). Disregarding the different illumination settings, the results for the first two exemplars ("woodwall" and "animalskin") appear to be of very similar quality. Our result for the "woodwall" texture seems to have more structure than Kopf et al.'s result, which looks rather smooth. Although our result for "animalskin" shows more variance in the size of the features, it better reflects the structures within the blue spots. On the other hand, the boundaries between texture features look sharper in Kopf et al.'s solid texture, which can also be seen in the right hand image, where features (the tomatoes) are more distinct and the green leaves are not suppressed as much. However, our technique is significantly faster. Including the initialization, we need about 6 minutes when using the half-reduced neighborhood and less than 9 minutes using the full $5 \times 5$ neighborhood. In contrast, Kopf et al. reported up to 90 minutes required for the synthesis. Another advantage of our proposed method over Kopf et al.'s solution is the fact that additional channels can be synthesized without affecting the running of the synthesis at all. In contrast, the cost of Kopf et al.'s method directly depends on the number of channels in the exemplar. Besides this, it has to be pointed out that Kopf et al.'s algorithm does not allow a straightforward implementation on the GPU, because a continuous update of the histogram is required.

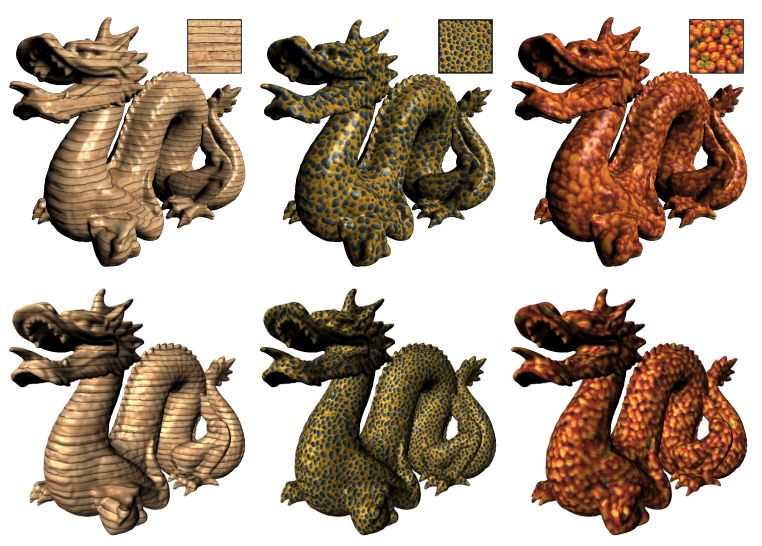

Figure 8: Comparison of 3D texture synthesis with Kopf et al.'s results. Top row: Our synthesis results, using the halfreduced neighborhood (left, right) and a full $5 \times 5$ neighborhood (center), respectively. Bottom row: Results of Kopf et al.'s algorithm with the same input exemplars and target resolution (from http://www.johanneskopf.de/ publications/solid/results/index.html.)

\section{CONCLUSIONS AND FUTURE WORK}

We presented a new and fast exemplar-based texture morphing algorithm for two, three and theoretically also higher dimensions as an extension of the pure 2D synthesis algorithm proposed in (Lefebvre and Hoppe, 2005; Lefebvre and Hoppe, 2006). Because our modifications obey the design principles of the original algorithm, our new contribution still allows an implementation on parallel stream-processing hardware. Even without hardware acceleration our current CPU-based implementation is already faster than comparable 3D synthesis methods. The steps of the original algorithm have been generalized to support morphing with arbitrary many exemplars and higher-dimensional synthesis. A more intuitive jitter function and a new compact neighborhood suitable for fast 3D synthesis have been introduced and its performance evaluated.

In the future we want to further improve the synthesis quality. Spatially varying scaling based on the dominant frequency of the exemplars could support the morphing to create better transitions between ex- 
emplars with structures of very different scale. Another interesting feature is the synthesis and morphing along time-varying vector and tensor fields.

\section{REFERENCES}

Ashikhmin, M. (2001). Synthesizing natural textures. In Proceedings of I3D '01, pages 217-226, New York, NY, USA. ACM Press.

Bar-Joseph, Z., El-Yaniv, R., Lischinski, D., and Werman, M. (2001). Texture mixing and texture movie synthesis using statistical learning. IEEE Transactions on Visualization and Computer Graphics, 7(2):120-135.

De Bonet, J. S. (1997). Multiresolution sampling procedure for analysis and synthesis of texture images. In Proceedings of SIGGRAPH '97, pages 361-368, New York, NY, USA. ACM Press.

Dischler, J.-M., Ghazanfarpour, D., and Freydier, R. (1998). Anisotropic solid texture synthesis using orthogonal 2d views. Computer Graphics Forum, 17(3):87-95.

Efros, A. A. and Freeman, W. T. (2001). Image quilting for texture synthesis and transfer. In Proceedings of SIGGRAPH '01, pages 341-346, New York, NY, USA. ACM Press.

Efros, A. A. and Leung, T. K. (1999). Texture synthesis by non-parametric sampling. In Proceedings of ICCV '99, pages 1033-1038, Washington, DC, USA. IEEE Computer Society.

Heeger, D. J. and Bergen, J. R. (1995). Pyramid-based texture analysis/synthesis. In Proceedings of SIGGRAPH '95, pages 229-238, New York, NY, USA. ACM Press.

Intel ${ }^{\circledR}$ Corporation (2008). Open Source Computer Vision Library. URL: http:// www.intel.com / technology / computing/opencv/index.htm [checked: 05/27/2008].

Jagnow, R., Dorsey, J., and Rushmeier, H. (2004). Stereological techniques for solid textures. ACM Transactions on Graphics (Proceedings of SIGGRAPH '04), 23(3):329-335.

Kopf, J., Fu, C.-W., Cohen-Or, D., Deussen, O., Lischinski, D., and Wong, T.-T. (2007). Solid texture synthesis from $2 \mathrm{~d}$ exemplars. ACM Transactions on Graphics (Proceedings of SIGGRAPH '07), 26(3):(2.1)-(2.9).

Kwatra, V., Essa, I., Bobick, A., and Kwatra, N. (2005). Texture optimization for example-based synthesis. ACM Transactions on Graphics (Proceedings of SIGGRAPH '05), 24(3):795-802.

Kwatra, V., Schödl, A., Essa, I., Turk, G., and Bobick, A. (2003). Graphcut textures: image and video synthesis using graph cuts. ACM Transactions on Graphics (Proceedings of SIGGRAPH '03), 22(3):277-286.

Lefebvre, S. and Hoppe, H. (2005). Parallel controllable texture synthesis. ACM Transactions on Graphics (Proceedings of SIGGRAPH '05), 24(3):777-786.

Lefebvre, S. and Hoppe, H. (2006). Appearance-space texture synthesis. ACM Transactions on Graphics (Proceedings of SIGGRAPH '06), 25(3):541-548.
Liu, Z., Liu, C., Shum, H.-Y., and Yu, Y. (2002). Patternbased texture metamorphosis. In Proceedings of $\mathrm{Pa}$ cific Graphics '02, page 184, Washington, DC, USA. IEEE Computer Society.

Matusik, W., Zwicker, M., and Durand, F. (2005). Texture design using a simplicial complex of morphable textures. ACM Transactions on Graphics (Proceedings of SIGGRAPH '05), 24(3):787-794.

Perlin, K. (1985). An image synthesizer. In Proceedings of SIGGRAPH '85, pages 287-296, New York, NY, USA. ACM Press.

Praun, E., Finkelstein, A., and Hoppe, H. (2000). Lapped textures. In Proceedings of SIGGRAPH 'O0, pages 465-470, New York, NY, USA. ACM Press.

Tong, X., Zhang, J., Liu, L., Wang, X., Guo, B., and Shum, H.-Y. (2002). Synthesis of bidirectional texture functions on arbitrary surfaces. In Proceedings of SIGGRAPH '02, pages 665-672, New York, NY, USA. ACM Press.

Tonietto, L. and Walter, M. (2005). Texture metamorphosis driven by texton masks. Computers \& Graphics, 29(5):697-703

Turk, G. (1991). Generating textures on arbitrary surfaces using reaction-diffusion. In Proceedings of SIGGRAPH '91, pages 289-298, New York, NY, USA. ACM Press.

Wei, L.-Y. (2002). Texture Synthesis by Fixed Neighborhood Searching. $\mathrm{PhD}$ thesis, Stanford University.

Wei, L.-Y. (2003). Texture synthesis from multiple sources. In SIGGRAPH '03: ACM SIGGRAPH 2003 Sketches \& Applications, pages 1-1, New York, NY, USA. ACM Press.

Wei, L.-Y. and Levoy, M. (2000). Fast texture synthesis using tree-structured vector quantization. In Proceedings of SIGGRAPH' '00, pages 479-488, New York, NY, USA. ACM Press.

Witkin, A. and Kass, M. (1991). Reaction-diffusion textures. SIGGRAPH Computer Graphics, 25(4):299_ 308.

Worley, S. (1996). A cellular texture basis function. In Proceedings of SIGGRAPH '96, pages 291-294, New York, NY, USA. ACM Press.

Zhang, J., Zhou, K., Velho, L., Guo, B., and Shum, H.Y. (2003). Synthesis of progressively-variant textures on arbitrary surfaces. ACM Transactions on Graphics (Proceedings of SIGGRAPH '03), 22(3):295-302. 